\title{
Fusion Imaging of Contrast-enhanced Ultrasound With CT or MRI for Kidney Lesions
}

\author{
THOMAS AUER ${ }^{1,2}$, ISABEL HEIDEGGER ${ }^{3}$, TOBIAS DE ZORDO $^{2,4}$, DANIEL JUNKER ${ }^{1,2}$, \\ WERNER JASCHKE ${ }^{2}$, FABIAN STEINKOHL ${ }^{2}$ and FRIEDRICH AIGNER ${ }^{2}$ \\ ${ }^{1}$ Department of Radiology, Landeskrankenhaus Hall, Hall in Tirol, Austria; \\ ${ }^{2}$ Department of Radiology, Medical University Innsbruck, Innsbruck, Austria; \\ ${ }^{3}$ Department of Urology, Medical University Innsbruck, Innsbruck, Austria; \\ ${ }^{4}$ Department of Radiology, Brixana Private Clinic, Brixen, Italy
}

\begin{abstract}
Aim: To evaluate the feasibility of ultrasound (US) computed tomography (CT) or magnetic resonance imaging (MRI) fusion imaging (FI) for localization and assessment of kidney lesions. Materials and Methods: Twenty-eight patients with kidney lesions previously detected on CT or MRI were included in this retrospective study. All 28 patients with kidney lesions, which were indefinable (42.9\%) or hard to localize (57.1\%) on gray-scale US alone, underwent FI of US with CT/MRI datasets. In 23 (82\%) patients with indeterminate kidney lesions, FI including contrast-enhanced US was conducted. Results: FI was successfully performed in 25 out of 28 (89.3\%) patients. FI with contrast-enhanced US was able to clarify the previously detected kidney lesions in 21 out of 23 patients (91.3\%). Conclusion: FI is a feasible technique for localizing kidney lesions that are hard to define by grayscale US alone and the additional application of contrast-enhanced US is useful in clarifying indeterminate CT or MRI findings.
\end{abstract}

This article is freely accessible online.

Data were handled according to the World Medical Association Declaration of Helsinki (59th WMA Assembly, Seoul, 2008). Institutional Review Board approval was further granted by means of a general waiver for studies with retrospective data analysis (Ethikkommission, Med. Univ. Innsbruck; 2009-02-20).

Correspondence to: Friedrich Aigner, MD, Assistant Professor of Radiology, Department of Radiology, Medical University Innsbruck, Anichstrasse 35, 6020 Innsbruck, Austria. E-mail: Friedrich.Aigner@tirol-kliniken.at

Key Words: Fusion imaging, ultrasound, contrast-enhanced ultrasound, computed tomography, magnetic resonance imaging, kidney.
Gray-scale ultrasound (US) is a widely used imaging technique for initial evaluation, treatment planning and characterization of kidney lesions, but establishing a final diagnosis is often impossible (1). If a kidney lesion shows similar echogenicity to renal parenchyma, is of small size or in a difficult anatomic location, initial conventional gray-scale US can fail to detect the lesion of interest $(2,3)$. In addition, the exact localization of a lesion by US can be challenging and time-consuming if multiple, adjacent and similarly shaped lesions are located within one kidney. In order to improve the diagnostic reliability of US and combine the advantages of different modalities, image fusion systems have been developed. Fusion imaging (FI) of US with computed tomography (CT) or magnetic resonance imaging (MRI) is able to merge real-time US with previously acquired CT or MRI datasets and can help localize or detect lesions or targets with poor conspicuity on conventional gray-scale US (4-8). After a complex kidney lesion has been definitively localized by gray-scale US, one should not rely on gray-scale US findings alone in the differentiation of this lesion because determination of contrast enhancement (CE) is a crucial factor in differentiating surgical from nonsurgical renal masses (9). Contrast-enhanced ultrasound (CEUS) offers excellent discriminative abilities in characterization of complex cystic lesions and is even more sensitive than CT in blood flow detection of hypovascular lesions $(10,11)$. In this study, we evaluated the feasibility of FI of (CE)US with CT or MRI to localize sonographically challenging kidney lesions and to further assess indeterminate CT/MRI findings by FI with CEUS.

\section{Materials and Methods}

Patients. Twenty-eight consecutive patients undergoing FI at our department were retrospectively analyzed in this study. All patients underwent solitary gray-scale US as well as FI with US/CT or US/MRI. If a kidney lesion was described as indeterminate in the previous CT or MRI, FI was additionally combined with CEUS. Exclusion criteria 
were age $<18$ years, unstable coronary heart disease and cardiac pacemaker. A kidney lesion with $>20 \mathrm{HU}$ in an unenhanced CT scan was defined as a lesion with high attenuation $(9,12)$. Kidney lesions that showed an increased attenuation in a range of 10-20 HU after application of contrast agent were defined as borderline CE lesions (9, 13). Data were handled according to the World Medical Association Declaration of Helsinki (59th WMA Assembly, Seoul, 2008). Institutional Review Board approval was further granted by means of a general waiver for studies with retrospective data analysis (Ethikkommission, Med. Univ. Innsbruck; 2009-02-20).

Gray-scale US and FI. All US examinations were performed on an ultrasonic device capable of FI (LOGIQ E9/GE) and a 1-5 MHz transducer (C1-5D/GE). For FI, an electromagnetic transmitter, which is placed near the area of interest, is required. Furthermore, electromagnetic sensors are attached to the transducer. The electromagnetic transmitter emits an electromagnetic field and the sensors attached to the transducer register the position of the transducer in this field. Previously recorded three-dimensional datasets can be shown simultaneously with real-time US on the screen of the US device. Firstly, the previously acquired patientrelevant CT or MRI datasets were loaded from the local database onto the ultrasonic device. In the next step, plane registration, in which the transducer is held parallel to the previously acquired cross-sectional slices, was used. Point registration, in which a specific anatomical point is first marked in real time and then synchronized with the cross-sectional dataset, was used for fine adjustments. After registration and image fusion, simultaneous realtime navigation through both gray-scale US images and CT/MRI datasets can be performed (3).

Fusion imaging with CEUS. In 23 patients, the indeterminate kidney lesion was further evaluated using both FI and CEUS. CEUS was performed after intravenous bolus injection of $1.2 \mathrm{ml}$ of SonoVue (Braccom, Milan, Italy) in the antecubital vein, followed by a $5 \mathrm{ml}$ flush of saline $(0.9 \%$ sodium chloride). In four patients, a second bolus of SonoVue was applied resulting in $2.4 \mathrm{ml}$ for a repeated evaluation. For CEUS scanning, a low mechanical index (mechanical index $<0.1$, temporal resolution between 10 and 13 frames per second) was used. Video clips with CEUS and FI were recorded and stored in the local database.

Interpretation of fusion imaging findings. FI examination was rated as successful if the real-time US of the kidney was correctly synchronized with the cross-sectional datasets. Based on CEUS, cystic kidney lesions were classified according to the established Bosniak classification for CEUS or as solid masses (14). If a lesion showed the exact same post-contrast performance as renal parenchyma, the lesion was rated as a pseudotumor (15). Histology or follow-up US were used as reference standards.

Statistical analysis. Descriptive statistics for feasibility of FI and kidney lesion characterization by FI-CEUS were performed. Patient characteristics and lesion size were summarized with frequencies and percentages and with mean and range.

\section{Results}

Twenty-eight patients with a mean age of $58.1( \pm 12.3)$ years (seven female and 21 male) were evaluated all patients were evaluated with FI (US and CT/MRI) and 23/28 (82.1\%) patients with FI and additional CEUS. Twenty-six CT datasets and two MRI datasets for FI were used (Table I).

Indications for fusion imaging. In 16 out of 28 patients $(57.1 \%)$, FI was performed because it was not possible to clearly localize the lesion of interest due to multiple and directly adjacent similar lesions within one kidney. In 12 out of 28 patients $(42.9 \%)$, the kidney lesions were solitary or at least isolated but indefinable by gray-scale US alone due to small lesion size, isoechogenicity (Figure 1) and/or a sonographically difficult anatomical location near the apical upper pole.

Feasibility of fusion imaging. FI was performed successfully in 25 out of $28(89.3 \%)$ patients. In three out of $28(10.7 \%)$ patients, FI failed and lesions were not confidently detected due to general US limitations, namely insufficient patient compliance with small lesion size in one patient $(6 \mathrm{~mm})$ and obesity in two patients.

Indications for FI and CEUS. CEUS was applied in 23 patients. In two patients, CEUS was not conducted despite successful image fusion because only size control was necessary and in three patients CEUS was not conducted because the lesion of interest could not be localized. Regarding these 23 patients, indications for FI with CEUS were as follows (see also Table I): (i) Insufficient CT protocol without non enhanced computed tomography (NECT) and a high attenuation kidney lesion in seven $(30.4 \%$ ) patients (Figure 2), (ii) borderline CE in CT in 10 (43.5\%) patients, (iii) non-conclusive CT vs. MRI studies in three $(13 \%)$ patients, (iv) non-diagnostic MRI in two $(8.7 \%)$ patients (Figure 3), (v) CEUS for follow-up in one patient.

Findings of FI with CEUS. In 21 out of 23 patients (91.3\%), CEUS was able to clarify the previously detected CT/MRI findings (see Table I). In two patients, FI with CEUS failed when compared to the histological reference standard: in one patient vascularization of a histologically proven papillary renal cell carcinoma (RCC) in an atrophic kidney could not be detected with CEUS, and in the other patient a histologically proven lipid-poor angiomyolipoma was diagnosed as a potentially malignant (partially cystic/Bosniak IV) lesion.

\section{Discussion}

In the present study, we showed that FI of US with CT/MRI is a feasible technique for localizing challenging kidney lesions. FI were performed successfully in the majority $(89.3 \%)$ of patients in which either a kidney lesion of interest could not definitively be localized due to multiple and directly adjacent similar lesions, or an indeterminate kidney lesion was solitary 
Table I. Lesion characteristics, indications for fusion imaging (FI) and contrast-enhanced ultrasound (CEUS), CEUS results, and final diagnosis.

\begin{tabular}{|c|c|c|c|c|c|}
\hline Patient & $\begin{array}{l}\text { Lesion size } \\
\quad(\mathrm{mm})\end{array}$ & $\begin{array}{c}\text { Indication } \\
\text { FI }\end{array}$ & $\begin{array}{l}\text { Indication for } \\
\text { CEUS }\end{array}$ & $\begin{array}{l}\text { CEUS } \\
\text { report }\end{array}$ & $\begin{array}{c}\text { Final } \\
\text { diagnosis }\end{array}$ \\
\hline 1 & 15 & Multiple/adjacent lesions & Insufficient $\mathrm{CT}$ protocol & Still indeterminate & Papillary RCC \\
\hline 2 & 10 & Indefinable by grayscale US alone & Insufficient $\mathrm{CT}$ protocol & Benign, post-ischemic scar & Benign \\
\hline 3 & 12 & Indefinable by grayscale US alone & Insufficient $\mathrm{CT}$ protocol & Benign, renal medulla & Benign \\
\hline 4 & 20 & Multiple/adjacent lesions & Insufficient $\mathrm{CT}$ protocol & B-II & Benign \\
\hline 5 & 33 & Multiple/adjacent lesions & Insufficient $\mathrm{CT}$ protocol & B-II & Benign \\
\hline 6 & 12 & Multiple/adjacent lesions & Insufficient $\mathrm{CT}$ protocol & B-II & Benign \\
\hline 7 & 15 & Multiple/adjacent lesions & Insufficient $\mathrm{CT}$ protocol & B-II & Benign \\
\hline 8 & 25 & Multiple/adjacent lesions & Borderline CE & Hypovascular tumor & Papillary RCC \\
\hline 9 & 15 & Indefinable by grayscale US alone & Borderline CE & Hypovascular tumor & Papillary RCC \\
\hline 10 & 12 & Indefinable by grayscale US alone & Borderline CE & Hypovascular tumor & Papillary RCC \\
\hline 11 & 15 & Multiple/adjacent lesions & Borderline CE & B-IIF & Follow-up >12 months: B-IIF \\
\hline 12 & 13 & Indefinable by grayscale US alone & Borderline CE & B-IIF & Follow-up >12 months: B-IIF \\
\hline 13 & 8 & multiple/adjacent lesions & Borderline CE & B-IIF & Follow-up >12 months: B-IIF \\
\hline 14 & 13 & Indefinable by grayscale US alone & Borderline CE & B-II & Benign \\
\hline 15 & 25 & Multiple/adjacent lesions & Borderline CE & B-II & Benign \\
\hline 16 & 10 & Multiple/adjacent lesions & Borderline CE & B-II & Benign \\
\hline 17 & 20 & Indefinable by grayscale US alone & Borderline CE & B-II & Benign \\
\hline 18 & 18 & Multiple/adjacent lesions & Non-conclusive CT and MRI & B-IV & Angiomyolipoma (lipid-poor) \\
\hline 19 & 8 & Multiple/adjacent lesions & Non-conclusive CT and MRI & B-IV & Papillary RCC \\
\hline 20 & 9 & Indefinable by grayscale US alone & Non-conclusive CT and MRI & B-II & Benign \\
\hline 21 & 20 & Multiple/adjacent lesions & Non-diagnostic MRI only & B-IIF & Follow-up >12 months: B-IIF \\
\hline 22 & 16 & Multiple/adjacent lesions & Non-diagnostic MRI only & B-IIF & Follow-up >12 months: B-IIF \\
\hline 23 & 16 & Multiple/adjacent lesions & Follow-up & Abscess, size reduction & Size reduction \\
\hline 24 & 14 & Multiple/adjacent lesions & - & Abscess, size reduction & Size reduction \\
\hline 25 & 7 & Indefinable by grayscale US alone & - & B-IV, stable size & Follow-up \\
\hline 26 & 20 & Indefinable by grayscale US alone & - & Not detected by FI & - \\
\hline 27 & 6 & Indefinable by grayscale US alone & - & Not detected by FI & - \\
\hline 28 & 16 & Indefinable by grayscale US alone & - & Not detected by FI & - \\
\hline
\end{tabular}

B: Bosniak classification (13); CT: computed tomography; MRI: magnetic resonance imaging; RCC: renal cell carcinoma.

but could not be sufficiently defined with gray-scale US alone. In the remaining $10.7 \%$ of the patients, the detection of a kidney lesion by FI failed due to general US limitations such as insufficient patient compliance or patient obesity.

For further classification of an indeterminate kidney lesion with US after cross-sectional imaging, definitive localization is essential. This may be easy in the case of simple cysts and solitary lesions, but in the case of single or multiple, adjacent and similarly shaped lesions with low conspicuity in grayscale US, detection is more complicated. In these challenging cases, FI is a useful tool and can help to definitively localize a lesion of interest. Helck et al. have already described a more accurate identification of kidney lesions with FI compared to US and CEUS alone (3). They also pointed out the advantage of FI especially for multifocal and small lesions, which is concordant with the findings in our study in which it was possible to detect lesions as small as $7 \mathrm{~mm}$ and localize them in the presence of multiple kidney lesions with FI (3). Similarly, it has already been shown that FI of US with crosssectional images leads in a higher confidence in liver-tumor identification with a lesser degree of operator dependency (4,
16-18). The approximately 10-minute additional operation time required for an experienced sonographer for FI set up and data import is reasonable regarding the added value in patients with indeterminate kidney lesions.

With rapid advances in diagnostic imaging, there has also been an increasing frequency of cross-sectional examinations in the past decades with an increasing number of incidentally detected kidney lesions (13). Sometimes the assessment of kidney lesions in cross-sectional imaging can be challenging or even impossible if there is hemorrhage, inadequate CT/MRI protocols, pseudo-enhancement or partial volume averaging effects (19-21). If adequate CT protocols are performed after an indeterminate kidney lesion is detected, additional investigations with cross-sectional imaging lead to high radiation exposure. Kidney lesions are best evaluated with multiphase CT protocols, but these are often lacking in cases of incidentally detected kidney lesions (22). Especially if a NECT scan is not available, contrast dynamics cannot be evaluated and suspicious kidney lesions with high attenuation content remain unclassified. One main advantage of FI for challenging kidney lesions is the subsequent evaluation of accurately localized lesions in 


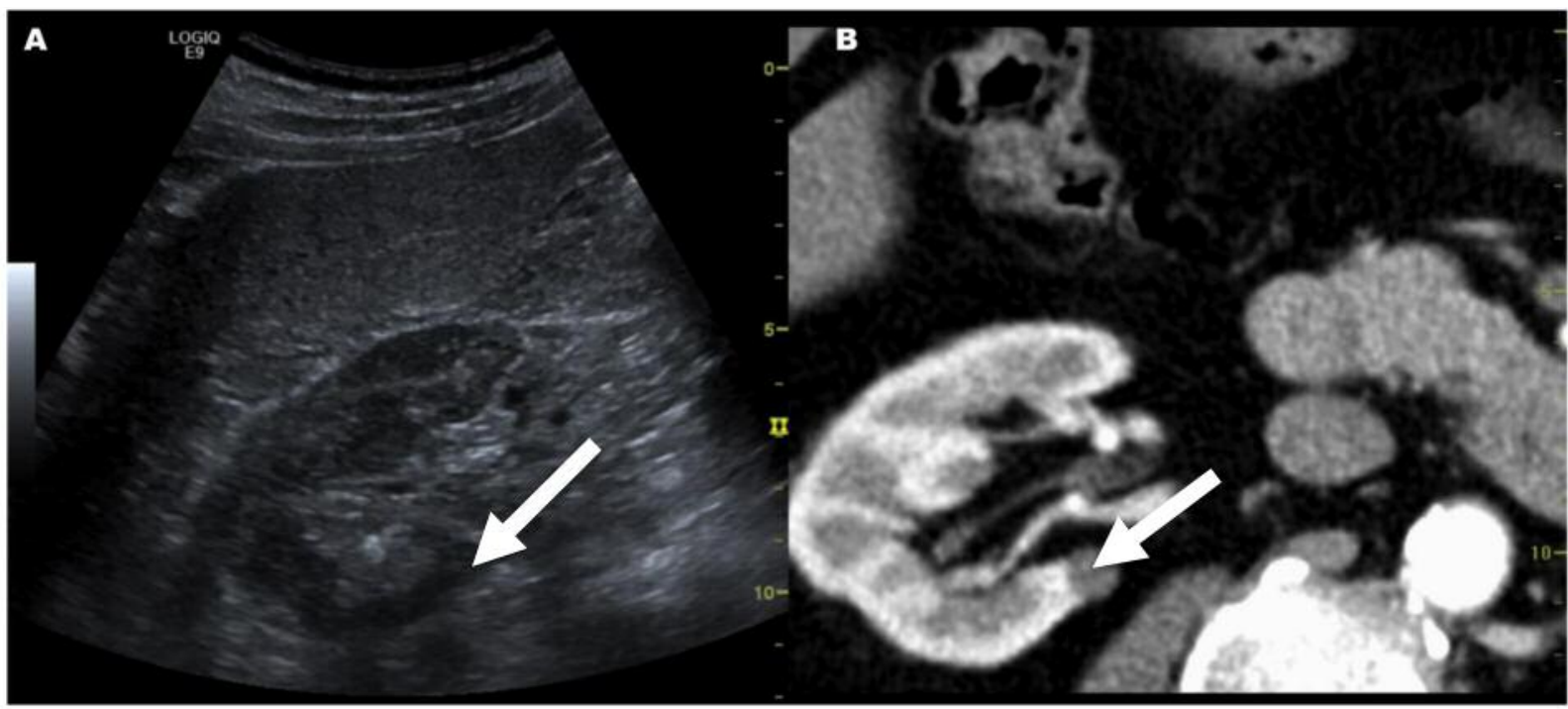

Figure 1. This image shows how fusion imaging is able to localize a kidney lesion which was not visible in gray-scale ultrasound (US) alone. After localization with fusion imaging, a solid tumor with contrast enhancement (Bosniak IV) was confirmed by contrast-enhanced US. Partial nephrectomy was performed and the histopathological finding was papillary renal cell cancer. A: In grayscale US, the kidney lesion was isoechoic (arrow) relative to the renal cortex and almost indefinable. B: Synchronized monophasic contrast-enhanced computed tomography showed indeterminate high-attenuation kidney lesion (arrow).

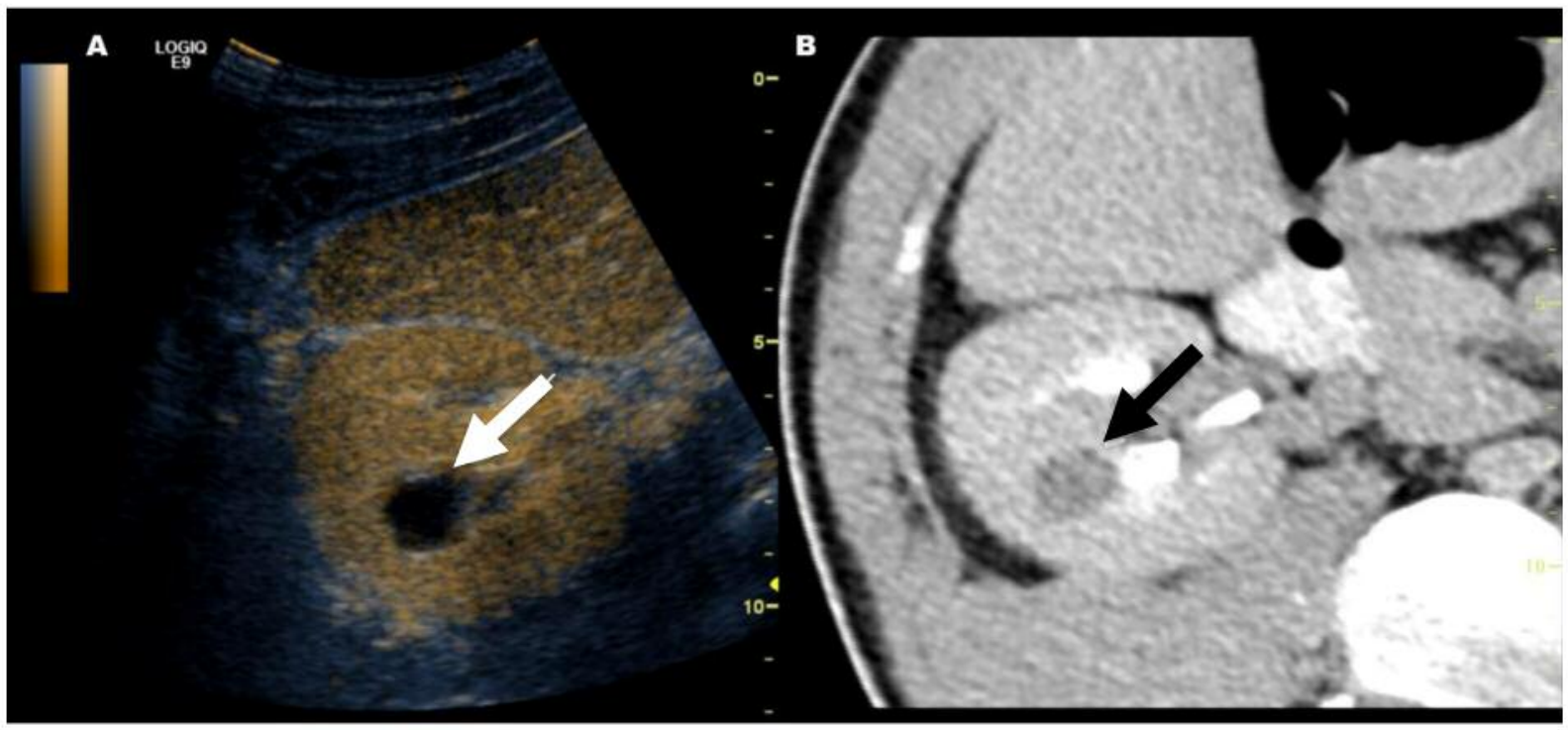

Figure 2. Fusion imaging of contrast-enhanced ultrasound (CEUS) and monophasic contrast-enhanced computed tomography (CT) in a patient with multiple similar kidney lesions (only one lesion is shown). A: Cystic lesion in CEUS with absence of contrast agent uptake on CEUS (arrow). $B$ : High-attenuation (>20 HU) kidney lesion (arrow) on a previously conducted monophasic contrast enhanced CT. After performing fusion imaging with the CT dataset, CEUS excluded contrast enhancement therefore the final diagnosis was Bosniak class II cyst.

combination with CEUS. Because gray-scale US alone has its limitations in the assessment of these indeterminate kidney lesions, we used CEUS as a cost-effective and radiation-free alternative for further classification $(11,23-26)$. With its high sensitivity for vascularization, CEUS is a valuable modality and can even be superior to CT in detecting blood flow in hypovascular lesions $(11,14)$. Besides characterization of cystic or solid renal masses, CEUS is indicated for the evaluation of 


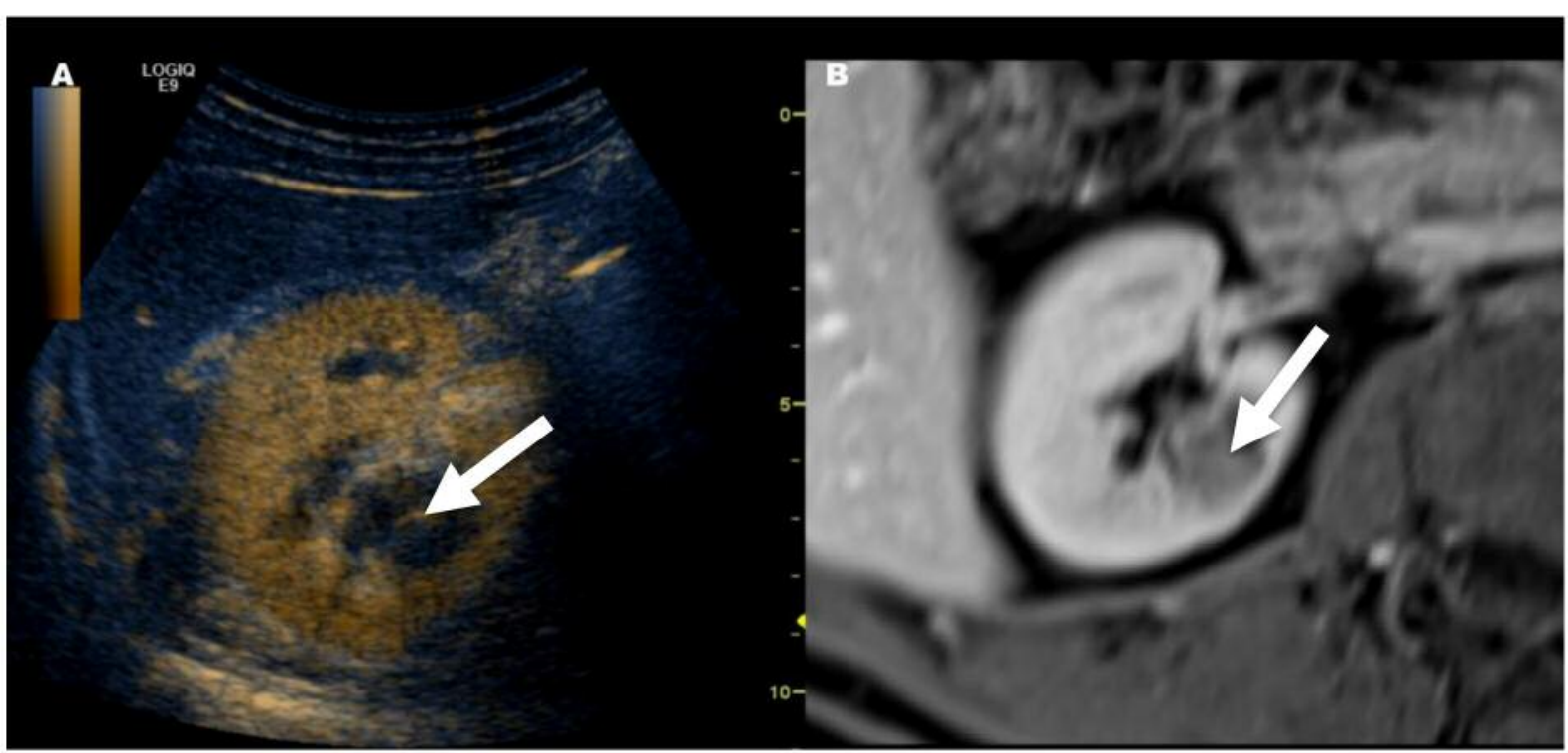

Figure 3. Downgrading of a Bosniak class III cyst on magnetic resonance imaging (MRI) to class IIf on contrast-enhanced ultrasound (CEUS). Follow-up confirmed the diagnosis of Bosniak class IIf. A: Image shows fusion imaging with CEUS; note that only septal enhancement (arrow) was visible. B Synchronized previously conducted contrast-enhanced MRI: Bosniak III cyst was diagnosed in MRI because more than septal enhancement was suspected.

renal infarctions, the differentiation between kidney tumors and anatomical variations, the follow-up of non-surgical complex masses, and the identification of clinically suspected renal abscesses in patients with complicated urinary tract infection (24). Using FI with CEUS, a definitive benign or malignant diagnosis was obtained in $91.3 \%$ patients that were evaluated with both FI and CEUS ( $n=25)$. In patients with Bosniak II cysts, not only insufficient CT protocols, but also borderline CE of the cystic lesions were found, which may be explained by pseudo-enhancement, partial volume effects and variability in HU measurement (i.e. beam hardening artifacts after spondylodesis). Furthermore, patients with hypersensitivity or anaphylactic reactions to iodine or gadolinium-based contrast agents and with challenging kidney lesions are suitable for FI with CEUS. In $20 \%$ of patients undergoing both FI and CEUS, a papillary RCC was histologically proven. Papillary RCC is the second most frequent RCC subtype and is known for its low degree of contrast enhancement in all post-contrast phases (27). The majority of papillary RCCs were detected with FI-CEUS, which highlights the sensitivity of FI with CEUS for hypovascular tumors, whereas in the previous CT examinations, all papillary RCCs only showed borderline CE of 10-20 HU. However, even with FI and CEUS, the detection of hypovascular tumors can be challenging. FI (-CEUS) further proved its value in the monitoring of two abscess formations and the identification of a pseudotumor $(15,24,28)$.

This study had several limitations. Because only patients with not clearly sonographically detectable kidney lesions were evaluated, we were only able to include a highly selected and, therefore, relatively low number of patients in this preliminary study. Nevertheless, good feasibility in this small population was shown for FI (-CEUS). Furthermore, the retrospective design of the study might have influenced our results, but the reader was blinded to the histological results. Moreover, only one radiologist with 10 years of experience in uroradiology determined candidates for FI, therefore interobserver agreement on inclusion criteria was not examined. In the retrospective design, it was not possible to evaluate interoperator agreement, but the stored real-time FI video clips with synchronized CT/MRI and (CE)US findings allowed for comprehensible retrospective image interpretation.

\section{Conflicts of Interest}

The Authors declare that there are no conflicts of interest in regard to this study and no commercial involvement.

\section{Conclusion}

FI of US and previously acquired CT or MRI datasets is a feasible imaging technique for detecting kidney lesions which are hard to define by gray-scale US alone. FI with CEUS can be utilized especially when the differentiation of a solid hypovascular lesion from a cystic lesion remains unresolved after CT/MRI imaging, or contrast-enhanced $\mathrm{CT} / \mathrm{MRI}$ is contraindicated. 


\section{References}

1 Helenon O, Correas JM, Balleyguier C, Ghouadni M and Cornud F: Ultrasound of renal tumors. Eur Radiol 11(10): 1890-1901, 2001.

2 Cokkinos DD, Antypa E, Kalogeropoulos I, Tomais D, Ismailos E, Matsiras I, Benakis S and Piperopoulos PN: Contrastenhanced ultrasound performed under urgent conditions. Indications, review of the technique, clinical examples and limitations. Insights Imaging 4(2): 185-198, 2013.

3 Helck A, D'Anastasi M, Notohamiprodjo M, Thieme S, Sommer W, Reiser M and Clevert DA: Multimodality imaging using ultrasound image fusion in renal lesions. Clin Hemorheol Microcirc 50(1-2): 79-89, 2012.

4 Lee MW, Rhim H, Cha DI, Kim YJ, Choi D, Kim YS and Lim HK: Percutaneous radiofrequency ablation of hepatocellular carcinoma: Fusion imaging guidance for management of lesions with poor conspicuity at conventional sonography. Am J Roentgenol 198(6): 1438-1444, 2012.

5 Nakano S, Kousaka J, Fujii K, Yorozuya K, Yoshida M, Mouri Y, Akizuki M, Tetsuka R, Ando T, Fukutomi T, Oshima Y, Kimura J, Ishiguchi $\mathrm{T}$ and Arai $\mathrm{O}$ : Impact of real-time virtual sonography, a coordinated sonography and MRI system that uses an image fusion technique, on the sonographic evaluation of MRI-detected lesions of the breast in second-look sonography. Breast Cancer Res Treat 134(3): 1179-1188, 2012.

6 Bercovich E, Leiderman M, Beck-Razi N, Gaitini D and Javitt M: Ultrasound-unenhanced CT fusion for detection and localization of ureteral stones. Am J Roentgenol 210(1): W8-W11, 2018.

7 Rubenthaler J, Paprottka KJ, Marcon J, Reiser M and Clevert DA: MRI and contrast enhanced ultrasound (CEUS) image fusion of renal lesions. Clin Hemorheol Microcirc 64(3): 457-466, 2016.

8 Theodore C, Levaillant JM, Capmas P, Chabi N, Skalli D, Vienet-Legue L, Haddad B, Fernandez H and Touboul C: MRI and ultrasound fusion imaging for cervical cancer. Anticancer Res 37(9): 5079-5085, 2017.

9 Israel GM and Bosniak MA: How i do it: Evaluating renal masses. Radiology 236(2): 441-450, 2005.

10 Barr RG, Peterson C and Hindi A: Evaluation of indeterminate renal masses with contrast-enhanced US: A diagnostic performance study. Radiology 271(1): 133-142, 2014.

11 Tamai H, Takiguchi Y, Oka M, Shingaki N, Enomoto S, Shiraki T, Furuta M, Inoue I, Iguchi M, Yanaoka K, Arii K, Shimizu Y, Nakata H, Shinka T, Sanke T and Ichinose M: Contrastenhanced ultrasonography in the diagnosis of solid renal tumors. J Ultrasound Med 24(12): 1635-1640, 2005.

12 Bosniak MA: The small (less than or equal to $3.0 \mathrm{~cm}$ ) renal parenchymal tumor: Detection, diagnosis, and controversies. Radiology 179(2): 307-317, 1991.

13 Silverman SG, Israel GM, Herts BR and Richie JP: Management of the incidental renal mass. Radiology 249(1): 16-31, 2008.

14 Ascenti G, Mazziotti S, Zimbaro G, Settineri N, Magno C, Melloni D, Caruso R and Scribano E: Complex cystic renal masses: Characterization with contrast-enhanced US. Radiology 243(1): 158-165, 2007.

15 Mazziotti S, Zimbaro F, Pandolfo A, Racchiusa S, Settineri N and Ascenti G: Usefulness of contrast-enhanced ultrasonography in the diagnosis of renal pseudotumors. Abdom Imaging 35(2): 241245, 2010.

16 Ewertsen C, Henriksen BM, Torp-Pedersen S and Bachmann Nielsen M: Characterization by biopsy or CEUS of liver lesions guided by image fusion between ultrasonography and CT, PET/CT or MRI. Ultraschall Med 32(2): 191-197, 2011.

17 Mauri G, Cova L, De Beni S, Ierace T, Tondolo T, Cerri A, Goldberg SN and Solbiati L: Real-time US-CT/MRI image fusion for guidance of thermal ablation of liver tumors undetectable with us: Results in 295 cases. Cardiovasc Intervent Radiol 38(1): 143-151, 2015.

18 Dong Y, Wang WP, Mao F, Ji ZB and Huang BJ: Application of imaging fusion combining contrast-enhanced ultrasound and magnetic resonance imaging in detection of hepatic cellular carcinomas undetectable by conventional ultrasound. J Gastroenterol Hepatol 31(4): 822-828, 2016.

19 Bae KT, Heiken JP, Siegel CL and Bennett HF: Renal cysts: Is attenuation artifactually increased on contrast-enhanced CT images? Radiology 216(3): 792-796, 2000.

20 Park BK, Kim B, Kim SH, Ko K, Lee HM and Choi HY: Assessment of cystic renal masses based on Bosniak classification: Comparison of CT and contrast-enhanced US. Eur J Radiol 61(2): 310-314, 2007.

21 Tappouni R, Kissane J, Sarwani N and Lehman EB: Pseudoenhancement of renal cysts: Influence of lesion size, lesion location, slice thickness, and number of mdct detectors. Am J Roentgenol 198(1): 133-137, 2012.

22 Van Der Molen AJ, Cowan NC, Mueller-Lisse UG, NolteErnsting CC, Takahashi S, Cohan RH; CT Urography Working Group of the European Society of Urogenital Radiology (ESUR): CT urography: Definition, indications and techniques. A guideline for clinical practice. Eur Radiol 18(1): 4-17, 2008.

23 Chen Y, Wu N, Xue T, Hao Y and Dai J: Comparison of contrastenhanced sonography with MRI in the diagnosis of complex cystic renal masses. J Clin Ultrasound 43(4): 203-209, 2014.

24 Piscaglia F, Nolsoe C, Dietrich CF, Cosgrove DO, Gilja OH, Bachmann Nielsen M, Albrecht T, Barozzi L, Bertolotto M, Catalano O, Claudon M, Clevert DA, Correas JM, D'Onofrio M, Drudi FM, Eyding J, Giovannini M, Hocke M, Ignee A, Jung EM, Klauser AS, Lassau N, Leen E, Mathis G, Saftoiu A, Seidel G, Sidhu PS, ter Haar G, Timmerman D and Weskott HP: The EFSUMB guidelines and recommendations on the clinical practice of contrast enhanced ultrasound (CEUS): Update 2011 on nonhepatic applications. Ultraschall in Med 33(1): 33-59, 2012.

25 Chang EH, Chong WK, Kasoji SK, Dayton PA and Rathmell WK: Management of indeterminate cystic kidney lesions: Review of contrast-enhanced ultrasound as a diagnostic tool. Urology 87: 1-10, 2016.

26 Gulati M, King KG, Gill IS, Pham V, Grant E and Duddalwar VA: Contrast-enhanced ultrasound (CEUS) of cystic and solid renal lesions: A review. Abdom Imaging 40(6): 1982-1996, 2015.

27 Egbert ND, Caoili EM, Cohan RH, Davenport MS, Francis IR, Kunju LP and Ellis JH: Differentiation of papillary renal cell carcinoma subtypes on CT and MRI. Am J Roentgenol 201(2): 347-355, 2013.

28 Fontanilla T, Minaya J, Cortes C, Hernando CG, Aranguena RP, Arriaga J, Carmona MS and Alcolado A: Acute complicated pyelonephritis: Contrast-enhanced ultrasound. Abdom Imaging 37(4): 639-646, 2012. 